\title{
Leukoplakia: A Comprehensive Review
}

\author{
Nitish Bhat ${ }^{1 *}$, Kalpna Thakur ${ }^{2}$, Lucky Jindal ${ }^{3}$, Hemwati Nandan $^{4}$, Farheen Arzoo ${ }^{5}$
}

\begin{abstract}
Leukoplakia is a potentially malignant disorder of the oral mucosa. It usually presents as a white patch in the oral cavity. The term leukoplakia represents a clinical entity and should preferably be used only for provisional diagnosis and not after histopathology. There are various terminologies and definitions associated with leukoplakia since past times. In this article, we have reviewed the literature to provide a comprehensive update on leukoplakia.
\end{abstract}

Keywords: Leukoplakia, Potentially malignant disorder, White Patch.

Asian Pac. J. Health Sci., (2020); DOI: 10.21276/apjhs.2020.7.2.8

\section{INTRODUCTION}

Leukoplakia is a frequently occurring potentially malignant lesion of the oral cavity. Hungarian dermatologist, Schwimmer in 1877, coined the term leukoplakia. It is derived from a Greek word Leucos which means white and Plakia, meaning a patch. ${ }^{[1]}$ Leukoplakia has been mentioned in the literature since 1969, but it was first defined by WHO in 1978, ${ }^{[2]}$ followed by other authors and even revised by WHO.

World Health Organisation (1978): Defined leukoplakia as a white patch or plaque that cannot be characterized clinically or pathologically as any other disease. ${ }^{[3]}$

$1^{\text {st }}$ International Conference on oral leukoplakia. Malmo, Sweden (1984): A white patch or plaque that cannot be characterized clinically or pathologically as any other disease and is not associated with any physical or chemical causative agent except the use of tobacco. ${ }^{[4]}$

International symposium. Uppsala, Sweden (1994): Oral leukoplakia is a predominantly white lesion of the oral mucosa that cannot be characterized as any other definable lesion; some oral leukoplakias will transform into cancer. ${ }^{[5]}$

Warnakulasuriya et al. (2007): Leukoplakia should be used to recognize white plaques of questionable risk having excluded (other) known diseases or disorders that carry no increased risk for cancer. ${ }^{[4]}$

Van der Waal 2012: "A predominantly white lesion or plaque of questionable behaviour having excluded, clinically and histopathologically, any other definable white disease or disorder". ${ }^{6]}$

It should be noted that leukoplakia is a clinical term only and the final diagnosis will be as per the histopathological evaluation. ${ }^{[7]}$

\section{Etiology}

Etiology for oral leukoplakia is multifactorial, but the tobacco in both forms seems to be associated in most of the lesions. The another frequently linked factor is alcohol but the use of alcohol as an independent aetiological factor in the development of oral leukoplakia, is still questionable. ${ }^{[8]}$

Other factors related to leukoplakia include Sanguinaria, Tertiary Syphilis, Deficiency of vitamins including vitamin A, B complex, C, E beta-carotene, Trauma, Viral Infection. ${ }^{1}$ Candidiasis is also considered an etiologic factor of leukoplakia but its possible role in the etiopathogenesis and in malignant transformation is still unclear. ${ }^{[9]}$

\section{Incidence}

In a study on large random samples, carried out in several geographic areas with various kinds of tobacco usage, the annual age-adjusted incidence rates of leukoplakia per 1000 population
${ }^{1}$ Registrar, Department of Oral Pathology, Indira Gandhi Govt. Dental College, Jammu, Jammu, and Kashmir, India.

${ }^{2}$ Lecturer, Oral Pathology and Microbiology, HP Govt. Dental College (IGMC), Shimla (HP), India

${ }^{3} \mathrm{MDS}$, Department of Paedodontics and Preventive Dentistry, JCD Dental College, Sirsa, Haryana, India

${ }^{4}$ Senior Resident, Department of Dentistry, All India Institute of Medical Science, Rishikesh, Uttarakhand, India.

${ }^{5}$ Dental Surgeon, Kolkata, India

Corresponding Author: Nitish Bhat, Registrar, Department of Oral Pathology, Indira Gandhi Govt. Dental College, Jammu, Jammu, and Kashmir, India., Email: nitishbhat04@gmail.com

How to cite this article: Bhat N, Thakur K, Jindal L, Nandan H, Arzoo F. Leukoplakia: A Comprehensive Review. Asian Pac. J. Health Sci., 2020; 7(2):33-35

Source of support: Nil

Conflict of interest: None

Received: 10/03/2020 Revised: 26/04/2020 Accepted: 05/05/2020

per year varied from 1.1-2.4 among men and from 0.2-1.3 among women; the prevalence varied from 0.2 to $4.9 \% .{ }^{[10]}$

In another study conducted by Pindborg et al. in 1967 on a large prospective study $(\mathrm{N}=10,000)$ in India and found that $3.28 \%$ of patients $(n=328)$ had leukoplakia. ${ }^{6}$ In a study done on the Swedish population, a prevalence rate of $3.6 \%$ was recorded. ${ }^{[11]}$

\section{Age and Gender}

The onset is usually seen after the age of 30 years of age, with peak incidence seen above the age of 50 years. The male to female ratio mainly depends on the geographic distribution of the disease but a strong male predominance in different parts in India, to almost $1: 1$ in the Western world. ${ }^{[8]}$

\section{ClinicalTypes}

According to WHO 19805[12]

Homogeneous leukoplakia has been defined as a predominantly white lesion of uniform flat, thin appearance that may exhibit shallow cracks and has a smooth, wrinkled or corrugated surface with a constant texture throughout.

Non-homogeneous leukoplakia has been defined as a predominantly white or white-and-red lesion (erythroleukoplakia) that may be irregularly flat, nodular or exophytic. The nodular lesions are characterized by white patches or nodules on an erythematous base.

O2020 The Author(s). This is an open access article distributed under the terms of the Creative Commons Attribution License (http://creativecommons.org/licenses/ by/4.0/), which permits unrestricted use, distribution, and reproduction in any medium, provided the original work is properly cited. 


\section{According to WHO (1998) $)^{[13]}$}

- Thin, smooth leukoplakia-Translucent thin grey soft flat plaques usually with sharply demarcated borders.

- Thick, fissured leukoplakia - 2/3 of white plaques has a distinctly white appearance, fissured and is leathery to palpation.

- Granular, verruciform leukoplakia - Lesions have surface irregularities of nodular or granular nature with verrucous appearance.

- Erythroleukoplakia - Lesion showing intermixed red and white areas.

\section{Warnakulasuriya et al. (2007) ${ }^{[14]}$}

Homogeneous leukoplakia - Homogeneous lesions are uniformly flat, thin, and exhibit shallow cracks of the surface keratin. The risk of malignant transformation is relatively low.

Non - Homogenous leukoplakia

- Speckled leukoplakia - Mixed, white and red, but retaining the predominantly white character

- Nodular leukoplakia - Small polypoid outgrowths, rounded red or white excrescences

- Verrucous-leukoplakia - Wrinkled or corrugated surface appearance.

Proliferative verrucous leukoplakia first described by Hansen (1985) is a subtype of verrucous leukoplakia which is characterized by an aggressive evolution, resistance to treatment, a multifocal appearance, higher degree of recurrence. ${ }^{[15]}$ It also has a high risk of transformation in oral carcinoma. ${ }^{[2]}$

Candidal Leukoplakia: It is an extremely chronic form of oral candidiasis seen as a firm, white, leathery plaques often found on the buccal mucosa, lips, and tongue. PAS staining is often used to differentiate it from other forms. Epithelial dysplasia is known to occur 4 to 5 times more frequently in candida leukoplakia than in leukoplakia. Carcinomatous change, also occur more frequently in this type. ${ }^{[1]}$

\section{Histology}

The histology of oral leukoplakia may vary from atrophy of the epithelium to hyperplasia with or without hyperkeratosis or may end into epithelial dysplasia or even carcinoma. Dysplasia reflects histological changes that are followed by the loss of uniformity or the architecture of the epithelial cells. The features suggesting dysplastic changes according to WHO criteria for epithelial dysplasia can be summed as follows:

\section{Architectural Changes ${ }^{[16]}$}

Irregular epithelial stratification, drop shaped rete ridges, loss of polarity of basal cells, abnormal superficial mitosis, premature keratinization in single cells (dyskeratosis), increased number of mitotic figures, Keratin pearls within rete ridges, loss of epithelial cell cohesion.

\section{Cellular Changes $^{[16]}$}

Abnormal variation in Nuclear size (anisonucleosis), abnormal variation in nuclear shape (nuclear pleomorphism), abnormal variation in cell shape (cellular pleomorphism), increased nuclearcytoplasmic ratio, abnormal variation in cell shape (anisocytosis) atypical mitotic figures, hyperchromasia, increased number and size of nucleoli.

Epithelial dysplasia, if present, may range from mild to severe. In some instances, carcinoma in situ and even squamous cell carcinoma are encountered histologically. It has been observed that the degree of epithelial dysplasia usually correlates with the age of the patient. ${ }^{[17]}$

\section{Diagnosis}

Clinical visual examination of the oral mucosa is the first and most frequent method for oral screening the patch on the oral mucosa followed by scrapping it with a sterile gauze piece. The experience and skill of the clinician are very important in such screening procedures, but it is not a method by which the risk level of the lesion can be calculated. ${ }^{[2]}$ So a combination of clinical history and histopathology of the lesion are important in making a final diagnosis.

There are various other adjunctive screening aids which have been used to assess the lesion these include vital staining with toluidine blue, brush biopsy examination, autofluorescence, chemiluminescence, and biomarker assessment (from saliva, serum, or exfoliated cells). ${ }^{[18]}$

In addition to this there are numerous molecular markers such as aberrant expression of p16INK4a and Ki-67, and loss of heterozygosity at $9 p$ and mutated TP $53^{[19,20]}$ but at present, there is no single marker or a set of markers which can be reliably used to predict malignant transformation of oral leukoplakia in an individual patient. ${ }^{[14]}$

\section{Management}

Management of leukoplakia is directed towards detecting any malignant transformation arising in the lesion. Management of leukoplakia starts with the cessation of tobacco use, followed by observation of the lesion. There are no strict guidelines that can be applied regarding the duration of follow-up examinations. In general, a long term follow-up examination is advised at 6-12 month intervals in patients who have not or not successfully been treated for their leukoplakia. Patients who, after treatment, remain disease-free for 3 years need perhaps no longer be followed-up. ${ }^{[21]}$

Various antifungal agents are given when Candida-associated leukoplakia is suspected. Other Management techniques include Chemoprevention- Retinoids, Vitamins (A, C, E), carotenoids, topical therapy- Bleomycin, Vitamin A, photodynamic therapy, surgical excision with or without grafting, electrocoagulation, cryosurgery and Laser surgery ${ }^{1}$ A Recent studies have reviewed the use of carbon dioxide laser for management of leukoplakia and have shown that the laser technique was reliable and reproducible with low morbidity. ${ }^{[6]}$

According to various studies, the recurrence of oral leukoplakia after surgical treatment has been reported in $10 \%^{[22]}$ to $15 \%^{[6]}$ cases. In Such recurrent lesions, the excision should be done, including $2 \mathrm{~mm}$ to $3 \mathrm{~mm}$ of the surrounding healthy margin. ${ }^{[23]}$ Patients having leukoplakia as a provisional diagnosis and a histopathologic diagnosis of SCC should be referred to an oncology department for further evaluation. ${ }^{[6]}$

\section{References}

1. Aggarwal N and Bhateja S "Leukoplakia. Potentially Malignant Disorder of Oral Cavity -a Review" Biomed J Sci \& Tech Res 2018;4(5):4219-4226.

2. Parlatescu I, Gheorghe C, Coculescu E, Tovaru S. Oral Leukoplakia - An Update a Journal of Clinical Medicine 2014; 9(1): 88-93.

3. Kramer IR, Lucas RB, Pindborg JJ, Sobin LH. Definition of leukoplakia of leukoplakia and related lesions: an aid to studies on oral precancer. Oral Surg Oral Med Oral Pathol. 1978;46:518-39.

4. Warnakulasuriya S, Johnson NW, van der Waal I. Nomenclature and classification of potentially malignant disorders of the oral mucosa. J Oral Pathol Med 2007; 36: 575-80. 
5. Axéll T, Pindborg JJ, Smith CJ, et al. Oral white lesions with special reference to precancerous and tobacco-related lesions: conclusions of an international symposium held in Uppsala, Sweden, May 18-21 1994. International Collaborative Group on Oral White Lesions. J Oral Pathol Med. 1996; 25:49-54.

6. Brouns E, Baart JA, Bloemena $E$, et al. - The relevance of uniform reporting in oral leukoplakia: Definition, certainty factor and staging based on experience with 275 patients. Med Oral Patol Oral Cir Bucal. 2012; 18756.

7. Villa A and Woo SB. Leukoplakia-A Diagnostic and Management Algorithm J Oral Maxillofac Surg 2017. 723-734.

8. Van der Waal I, Schepman KP, van der Meij EH, and Smeele LE. Oral Leukoplakia: a Clinicopathological Review. Oral Oncology Vol. 33, No. 5, pp. 291 301, 1997.

9. Kramer, I. R. H., Precancerous conditions of the oral mucosa. A computer-aided study. Annals of the Royal College of Surgeons, England, 1969, 45, 340- 356.

10. Gupta PC, Mehta FS, Daftary DK, et al., Incidence rates of oral cancer and natural history of oral precancerous lesions in a 10-year follow-up study of Indian villagers. Community Dental and Oral Epidemiology, 1980;8: 287-333.

11. Axell T.A prevalence study of oral mucosal lesions in an adult Swedish population. Odontologist Revy, 1976;27(36).

12. Gupta PC, Bhonsle RB, Murti PR., Daftary DL, Mehta FS and Pindborg JJ. An epidemiologic assessment of cancer risk in oral precancerous lesions in India with special reference to nodular leukoplakia. Cancer, 1989, 63, 22472252.

13. Neville BW, Day TA (2002) Oral Cancer and Precancerous Lesions. CA

14. Van der Waal I. Oral potentially malignant disorders: Is malignant transformation predictable and preventable? Med Oral Patol Oral Cir Bucal. 2014 Jul 1;19 (4):e386-910.

15. Van der Waal I, Reichart PA. Oral proliferative verrucous leukoplakia revisited. Oral Oncol 2008; 44:719-21.

16. Ranganathan K, Kavitha L. Oral epithelial dysplasia: lassification and clinical relevance in the risk assessment of oral potentially malignant disorders.Journal of Oral and Maxillofacial Pathology 2019 Jan Apr;23(1):19-27.

17. Vesper M., Schmelzle R., Ritter-Kr6h R. and Gfinzl HJ, Vergleichende Untersuchung histologisch gesicherter Leukoplakien im Cavum oris. Deutsche Zahndrztliche Zeitsehr, 1992, 47, 867-869.

18. Lingen MW, Kalmar JR, Karrison T, et al: Critical evaluation of diagnostic aids for the detection of oral cancer. Oral Oncol 44:10, 2008.

19. Nasser W, Flechtenmacher Chr, Holzinger D, Hofele Chr, Bosch FX. Aberrant expression of p53, p16INK4a and Ki-67 as basic biomarker for malignant progression of oral leukoplakias. J Oral Pathol Med. 2011;40:629-35.

20. Graveland AP, Bremmer JF, de Maaker M, Brink A, Cobussen P, Zwart M, et al. Molecular screening of oral precancer. Oral Oncol. 2013;49:1129-35.

21. Bouquot JE and Whitaker SB. Oral leukoplakia--ration- ale for diagnosis and prognosis of its clinical subtypes or "phases". Quintessence International, 1994, 25, 133-140.

22. Lodi G, Porter S. Management of potentially malignant disorders: evidence and critique. J Oral Pathol Med 2008; 37:63-69.

23. Mogedas-Vegara A, Hueto-Madrid JA, Chimenos-Kustner E et al: Oral leukoplakia treatment with the carbon dioxide laser: A systematic review of the literature. J Craniomaxillofac Surg 2016; 44: 331. 